\title{
Non-identical neural mechanisms for two types of mental transformation: event-related potentials during mental rotation and mental paper folding
}

\author{
B. Milivojevic, B.W. Johnson*, J.P. Hamm, M.C. Corballis \\ Department of Psychology, University of Auckland, Private Bag 92019, Auckland, New Zealand
}

Received 5 April 2002; received in revised form 29 January 2003; accepted 4 February 2003

\begin{abstract}
Reaction times, accuracy and 128-channel event-related potentials (ERPs) were measured from 14 normal, right-handed subjects while they performed two different parity-judgment tasks that require transformations of mental images: a relatively simple task requiring a single transformation (mental letter rotation), and a more complex task involving a coordinated sequence of transformations (mental paper folding). Reaction times increased monotonically with larger angular displacements from the upright (for mental rotation) and with number of squares carried (for mental paper folding). Both the tasks resulted in amplitude modulation of an approximately $420-700 \mathrm{~ms}$ latency ERP component at parietal electrodes. Scalp topographies indicated that right parietal cortex was activated during mental rotation, but bilateral parietal regions were activated during mental paper folding. Our results support the notion of a right hemispheric superiority for tasks involving simple, single mental rotations, but indicate greater involvement of the left hemisphere when a more complex sequence of transformations are required. This task-dependent lability of hemispheric function may account for some of the inconsistent results reported by previous neuroimaging and electrophysiological studies.
\end{abstract}

(C) 2003 Elsevier Science Ltd. All rights reserved.

Keywords: Electroencephalography; Visual event-related potentials; Mental rotation; Mental paper folding; Topography

\section{Introduction}

Mental imagery involves the generation and manipulation of mental representations in the absence of appropriate sensory input. The most extensively studied mental-image manipulation is mental rotation, which is the ability to imagine visual shapes rotated to an orientation other than that in which they appear. Since reaction times are longer for larger angles of misorientation, it has been proposed that mental representations of objects are rotated through a trajectory in much the same manner that physical objects are rotated. Assuming a constant rate of mental rotation, it would thus take longer to traverse larger angular displacements (Cooper \& Shepard, 1973; Shepard \& Metzler, 1971).

Cooper and Shepard (1973) introduced a simple paradigm for studying mental rotation in which they presented asymmetric alphanumeric characters and required subjects to decide if each was normal or mirror-reversed (parity-judgment). These authors reasoned that if such characters are internally represented in their normal upright

\footnotetext{
* Corresponding author. Tel.: +64-9-373-7599x88522; fax: +64-9-373-7450

E-mail address: b.johnson@auckland.ac.nz (B.W. Johnson).
}

(canonical) positions, misoriented images would have to be mentally-rotated in order to perform the discrimination. Since the normal and mirror-reversed characters possess the same features, this task ensured that the discrimination could not be accomplished by alternative feature-based strategies that are independent of orientation. Indeed, the observed RT functions were entirely consistent with a mental rotation strategy, showing a sharp increase as a function of the angular departure of characters from their upright positions. These RT functions have been extensively replicated in parity-judgment tasks involving the discrimination of not only mirror-imaged characters, but also of left and right hands (Cooper \& Shepard, 1975) of the mirror-image polygons (Ely, 1982), and of left- and right-facing naturalistic objects (Jolicoeur, 1985). Further, these RT functions are unique to parity judgment tasks: to our knowledge the only comparable phenomenon is the increased RTs obtained when subjects are required to identify rotated characters (Corballis, Zbrodoff, Shetzer, \& Butler, 1978; White, 1980) or naturalistic objects (Jolicoeur, 1985). However, these tasks typically produce shallower RT slopes and a "dip" at $180^{\circ}$, and it is debatable whether mental rotation is actually involved (see Jolicoeur, 1990; Murray, 1997) for more detailed treatments of this issue). Thus, there is good evidence 
that the sharp RT functions elicited by parity judgments of misoriented shapes are a unique "behavioral signature" of mental rotation.

If mental rotations are analogous to physical rotations, then this type of processing must be quite different from other types of computations that occur in the brain. The observation that reaction-time functions for mental rotation are continuous (such that intermediate RTs will be obtained for intermediate angles) indicates that it is implemented with an analogue type of code. If the computations involved were digital (for example), then there is no reason to expect processing time to increase with angular orientation in this fashion. Thus, mental rotation seems to require a code that is fundamentally different from that required by other important mental processes such as language (c.f. Corballis, 1997).

There is considerable interest in how a mental process with analogue properties might be implemented in the brain. Two lines of neurophysiological evidence are particularly pertinent to this issue. First, Georgopoulos (1995), Georgopoulos and Pellizzer (1995), Georgopoulos, Lurito, Petrides, Schwartz, and Massey (1989), and Georgopoulos, Taira, and Lukashin (1993) have demonstrated that an imagined movement is represented by an analogue sweep of the neuronal population vector in primary motor cortex of monkeys. Second, there is substantial convergent evidence from neuropsychological studies (e.g. Farah \& Hammond, 1988), functional brain imaging (e.g. Alivisatos \& Petrides, 1997; Harris et al., 2000; Kosslyn, Digirolamo, Thompson, \& Alpert, 1998; Tagaris et al., 1996, 1997), and electrophysiological recordings (Peronnet \& Farah, 1989; Stuss, Sarazin, Leech, \& Picton, 1983) that mental rotation also draws upon processes in the parietal lobe of the brain. Thus, current evidence supports the feasibility of analogue processing at the neuronal level, and points to the parietal lobe as a particularly important zone for the computations underlying mental rotation.

Mental rotation may also be more dependent on processes in the right than in the left hemisphere. One commissurotomized person, for example, proved initially unable to mentally rotate letters or simple stick figures when they were presented to his right visual field and, thus to the left hemisphere, but was able to do so when they were presented to his left visual field and right hemisphere (Corballis \& Sergent, 1989). Although his left hemisphere gained some proficiency in later testing, it remained inferior to the right, and may have adopted strategies other than analogue rotation. In support of this observation, Farah and Hammond (1988) reported that patients exhibit a deficit in mental rotation following right, but not left, parietal damage, and Harris et al. (2000), in a PET study, found selective activation in the right parietal lobe during a mental-rotation task.

However, not all studies have shown the right hemisphere to be critical for mental rotation. Mehta and Newcombe (1991) reported that patients with lesions restricted to the left hemisphere show deficits on mental-rotation tasks. Contrary to the evidence of Harris et al. (2000), other brain-imaging studies have suggested bilateral parietal involvement rather than exclusive right-hemisphere involvement (e.g. Kosslyn et al., 1998; Tagaris et al., 1996, 1997). These studies, however, used a mental-rotation task similar to that devised by Shepard and Metzler (1971), in which subjects rotate unfamiliar three-dimensional torus shapes, whereas Harris et al. (2000) used the simpler Cooper and Shepard (1973) task, in which subjects rotate familiar alphanumeric characters in two-dimensional space. There is some evidence that the Shepard-Metzler figures are rotated in piecemeal fashion (Bethell-Fox \& Shepard, 1988; Just \& Carpenter, 1985), and it may be this aspect, rather than the rotation component itself, that favors the left hemisphere (cf. Corballis, 1991). More generally, the left hemisphere may be increasingly engaged in spatial performance as the complexity of the task increases (De Renzi, 1978; McGuinness \& Bartell, 1982).

This suggests that Cooper and Shepard's task (1973), in which the subjects are presented with asymmetrical normal or mirror-reversed forms of alphanumeric characters presented at varying orientations from upright and are required to determine whether these characters are normal or mirror-reversed, may be a better measure of pure mental rotation, uncontaminated by piecemeal processing. Yet even this task has failed to yield consistent results in neuroimaging studies. Harris et al. (2000) did find right-parietal activation, but Alivisatos and Petrides (1997) found activation in the left inferior and posterio-superior parietal cortices and Tagaris et al. found bilateral activation of parietal areas (Tagaris et al., 1997). Thus, the issue of whether mental rotation processes are lateralised to one hemisphere or the other has not yet been resolved.

A difficulty with studies using PET or fMRI is that these techniques have relatively poor temporal resolution. Typically, PET involves averaging activity over a minute or more while most fMRI paradigms yield a temporal resolution of approximately $6 \mathrm{~s}$. As a consequence these techniques are likely to provide a temporally smeared image of sensory, perceptual, cognitive and motor elements of a given mental-rotation task. In the present study, we attempt to circumvent this problem by using EEG to locate the mental-rotation component in time. The utility of EEG in this context derives from the fact that mental rotation has well-established electrophysiological markers. Peronnet and Farah (1989) measured event-related potentials (ERPs) in response to misoriented alphanumeric characters, and found a negative going modulation of a $400-800 \mathrm{~ms}$ latency component at parietal electrodes. This modulation was correlated with angular departure of the stimuli from upright, suggesting that it was related to mental rotation itself. These findings have been extensively replicated in more recent research (e.g. Heil, Rauch, \& Hennighausen, 1998). In the present study, we used the mental-rotation task of Cooper and Shepard (1973), because this is uncontaminated by sequential, piecemeal processing, and as such provides a relatively pure measure of the mental rotation. We wished to characterize the spatial distribution of mental rotation ERPs 
using a high-density, 128-electrode montage, in order to address questions concerning hemispheric lateralization. Previous EEG studies have not employed more than about 50 electrodes.

We also wished to compare brain responses obtained in this relatively pure mental-rotation task to those obtained with a more complex mental transformation. Shepard and Feng (1972) devised a mental paper-folding task in which subjects must imagine re-folding the six interconnected squares representing an unfolded and flattened cube. Each individual fold can be performed by a simple mental rotation of a square (out of the picture plane), but multiple folds require a coordinated sequence of these transformations. As there is evidence that the left hemisphere is specialized for piecewise decomposition of shapes (see Corballis, 1991 for a review), we hypothesized that mental paper folding may invoke greater left hemisphere activity than simple mental rotation. To our knowledge, there have been no previous electrophysiological or neuroimaging studies of the Shepard and Feng (1972) mental paper-folding task.

\section{Method}

\subsection{Subjects}

Eighteen neurological normal subjects were recruited from students and faculty at the University of Auckland, New Zealand, and paid NZ\$20.00 for approximately $2.5 \mathrm{~h}$ of participation. All were right-handed as assessed by the Edinburgh Handedness Inventory (Oldfield, 1971) (mean rating of 88.57 , range from 80 to 100 ). The procedures were approved by the University of Auckland Human Subjects Ethics Committee. Four subjects were dropped from analysis due to ocular and/or movement artefact during recording. Thus, 14 subjects (seven females) were included in the final analysis. They ranged in age from 19 to 32 years, with a mean of 22.9 years.

\subsection{Visual displays}

The experiments were programmed on a Pentium II/200 computer. The stimuli were centered on a $15 \mathrm{in}$. flat-screen SVGA monitor with a resolution of 640 pixel $\times 480$ pixel. The subjects were seated at a distance of $57 \mathrm{~cm}$ from the display screen, and monitored continuously with a closed circuit video camera. TTL pulses generated via the parallel port of the display computer provided synchronization of stimulus events with EEG acquisition. Millisecond timing routines for the visual displays were from Hamm (2001). Synchronization with the raster scan was achieved with the method of Heathcote (1988) with further synchronization to the top of the stimulus achieved as suggested by Hamm (2001).

The stimuli for the mental-rotation task consisted of six uppercase alphabetic characters (F, G, J, L, P and R). These

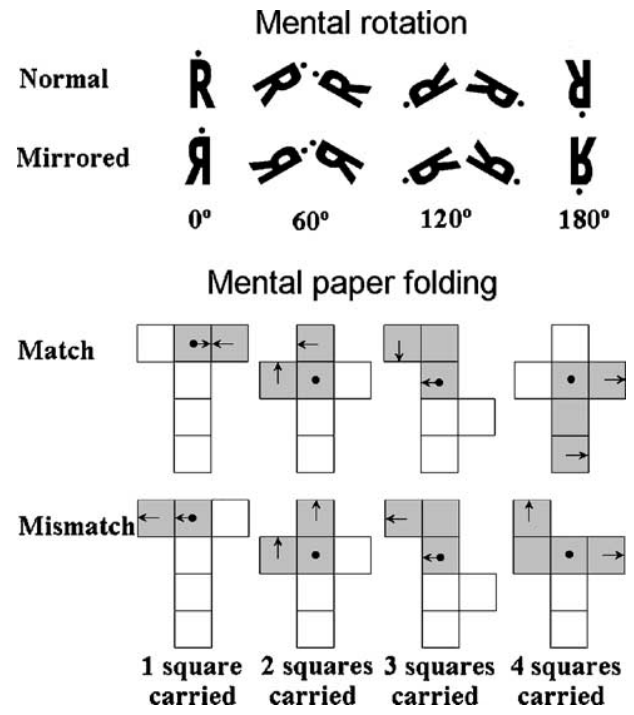

Fig. 1. Examples of stimuli for mental rotation and mental paper-folding tasks. Grey shading on paper-folding stimuli shows base square and folding squares for each subconfiguration (experimental stimuli were not shaded).

letters subtended a vertical visual angle of $2.71^{\circ}$. Each letter was presented in both a normal and mirror-reversed form at angular departures of $0,60,120,180,240$, and $300^{\circ}$ clockwise from the upright. ${ }^{1}$ A small black dot was displayed at the true top of each character to provide an explicit cue to aid in rotating the figure (see Fig. 1). The stimuli were presented as black letters on a grey background.

The stimuli for mental paper folding consisted of black outline drawings of six squares joined together, representing faces of an unfolded cube. A small black dot was presented in the middle of one of the squares marking it as the base of the cube. Two small arrows were presented on two of the squares, each centered on one of the sides of the square. Half of the displays were "match" cases in which the arrows would meet if the squares were folded up into a cube. The other half of the displays were "mismatch" cases in which the two arrows could not be made to meet by folding. The stimuli were classified into types according to the total number of squares carried along for each fold in order for the match-mismatch decision to be made (see Shepard $\&$ Feng, 1972 for more detail). The displays were generated so that there were four match and four mismatch examples of the four basic types of 1-4 squares carried. This resulted

\footnotetext{
${ }^{1}$ It was intended that the letters presented at 0 and $180^{\circ}$ would be presented twice as often as letters at $60,120,240$ and $300^{\circ}$, since the 60 and $120^{\circ}$ orientations were to be treated as equivalent to 300 and $240^{\circ}$, respectively. However, due to a programming error which was not discovered until after completion of the data collection, the letters at $120^{\circ}$ orientations were presented twice as often as the letters at 60, 180, 240 and $300^{\circ}$ orientations. This resulted in 60:60:90:30 presentations for 0 , 60,120 and $180^{\circ}$ orientations, respectively. The data were compared to a series of comparable data sets from this laboratory and with published data from other laboratories, and we concluded that the unintended imbalance of conditions had no measurable effect on the behavioral or ERP results.
} 
in a total of 64 presentations per condition. The stimuli were presented against a uniform grey background and subtended a vertical visual angle of $8.48^{\circ}$. Fig. 1 illustrates all the subconfigurations used in the task and a corresponding example of each type of stimulus.

\section{3. $E E G$}

Recordings were carried out in an electrically-shielded Faraday room. Electrical Geodesics Inc. 128-channel $\mathrm{Ag} / \mathrm{AgCl}$ electrode nets (Tucker, 1993) were used, see Johnson and Hamm (2000) for a detailed description of electrode positions with gross head and brain anatomy. With this system, electrode locations are determined by the geometry of the sensor net. For convenience in discussion, those electrodes closest to standard positions are labeled with Ten-Twenty System labels (Jasper, 1958) but actual placement of any given electrode may be as far as $1-2 \mathrm{~cm}$ away from the designated position.

EEG was recorded continuously $(250 \mathrm{~Hz}$ sampling rate, $0.1-100 \mathrm{~Hz}$ analogue filter) with Electrical Geodesics Inc. amplifiers (200 M $\Omega$ input impedance) and acquisition software running on a Power Macintosh 9600/200 computer with a National Instruments PCI-1200 12 bit analogue-to-digital conversion card. Electrode impedances were below $50 \mathrm{k} \Omega$ (range of: $30-50 \mathrm{k} \Omega$ ), an acceptable level with the high input impedance amplifiers used in this EEG system (Ferree, Luu, Russell, \& Tucker, 2001). EEG was acquired using a common vertex $\left(C_{z}\right)$ reference.

\subsection{Procedure}

In total, there were 480 trials for the mental-rotation task and 512 trials for the mental paper-folding task. Each trial began with a $250-\mathrm{ms} 300-\mathrm{Hz}$ warning tone. One second later the visual stimulus appeared. In the mental-rotation task, the visual stimulus remained on the screen for a maximum of $4 \mathrm{~s}$ or until the subject responded, whichever was the sooner. If no response was made within the time limit the trial was omitted from further analysis. As we did not know in advance what range of RTs to anticipate for the mental paper-folding task, we did not initially impose a time-limit on trials (the stimulus remained on the screen until the subject responded); after data collection was complete, we eliminated trials with RTs greater than $6 \mathrm{~s}$ from analysis. There was a $500 \mathrm{~ms}$ delay after the response, or after the $4 \mathrm{~s}$ time-out in the mental-rotation task, before another tone signaled the end of the trial.

Subjects were instructed to avoid eye-blinks or other movements until the second tone sounded. To minimize movement artefact, they were instructed to keep their left hands in their laps, and to rest their index and middle fingers of their right hands on the keyboard.

In the mental-rotation task, subjects were required to press the " 1 " key if they judged the character to be normal, and the " 2 " key if they judged it to be mirrored. In the mental paper-folding task, they were to press the "1" key if they judged that the arrows in two of the squares of the unfolded cube would meet ("match") if the cube were folded up, and the "2" key otherwise ("mismatch"). In both the cases they were instructed to respond as quickly as possible without sacrificing accuracy.

\section{Results}

\subsection{Behavioral results}

\subsubsection{Mental-rotation task}

Fig. 2(A) shows the reaction times as a function of angle of orientation for normal and mirror-reversed letters. Overall, the reaction times for mirror-reversed letters were significantly longer than the reaction times for normal letters $(F(1,13)=15.19, P=0.002)$, by an average of $160 \mathrm{~ms}$. There was also a significant main effect of orientation $(F(3,39)=79.08, P<0.001)$, which was dominated by a linear trend $(F(1,13)=106.65, P<0.001)$, but the quadratic $(F(1,13)=27.98, P<0.001)$ and cubic $(F(1,13)=31.41, P<0.001)$ trends were also highly significant. The linear trend accounted for $87 \%$ of the variance, quadratic trend $11 \%$, and the cubic trend approximately $2 \%$. Reaction times for upright letters were $487 \mathrm{~ms}$ shorter than the reaction times for letters presented on an $180^{\circ}$ deflection from upright. The interaction between the two main effects did not approach significance $(F(3,39)<1)$.

Overall, the accuracy (Fig. 2(B)) for all responses was $95.22 \%$. There was no significant difference in accuracy between responses to normal and mirror-reversed letters $(F(1,13)<1)$. There was a significant main effect of orientation on accuracy $(F(3,39)=11.23, P<0.001)$. This effect consisted of significant linear $(F(1,13)=12.70, P=$ $0.003)$ and quadratic $(F(1,13)=9.43, P=0.009)$ trends, with the linear trend explaining $68 \%$ and the quadratic trend $32 \%$ of the variance. The maximal accuracy was achieved for the letters that were presented at $60^{\circ}$ deflection $(98.33 \%)$ and the minimum accuracy was achieved for the letters that were presented at $180^{\circ}(90.18 \%)$. The accuracy for $0^{\circ}$ was significantly different from accuracy for $180^{\circ}(P=0.032$ with Bonferroni correction) and the accuracy for $60^{\circ}$ was significantly different from accuracy for 120 and $180^{\circ}(P<$ 0.010 for both differences, with Bonferroni correction).

\subsubsection{Paper-folding task}

Fig. 2(C) shows the reaction times as a function of number of squares carried, for matching and non-matching shapes. The reaction times for mental paper folding revealed a significant difference between the matching and non-matching shapes $(F(1,13)=35.61, P<0.001)$. Responses to non-matching shapes were $215 \mathrm{~ms}$ longer than the responses to matching shapes. There was also a significant main effect of number of squares carried during each fold $(F(3,39)=47.21, P<0.001)$. The difference between the 

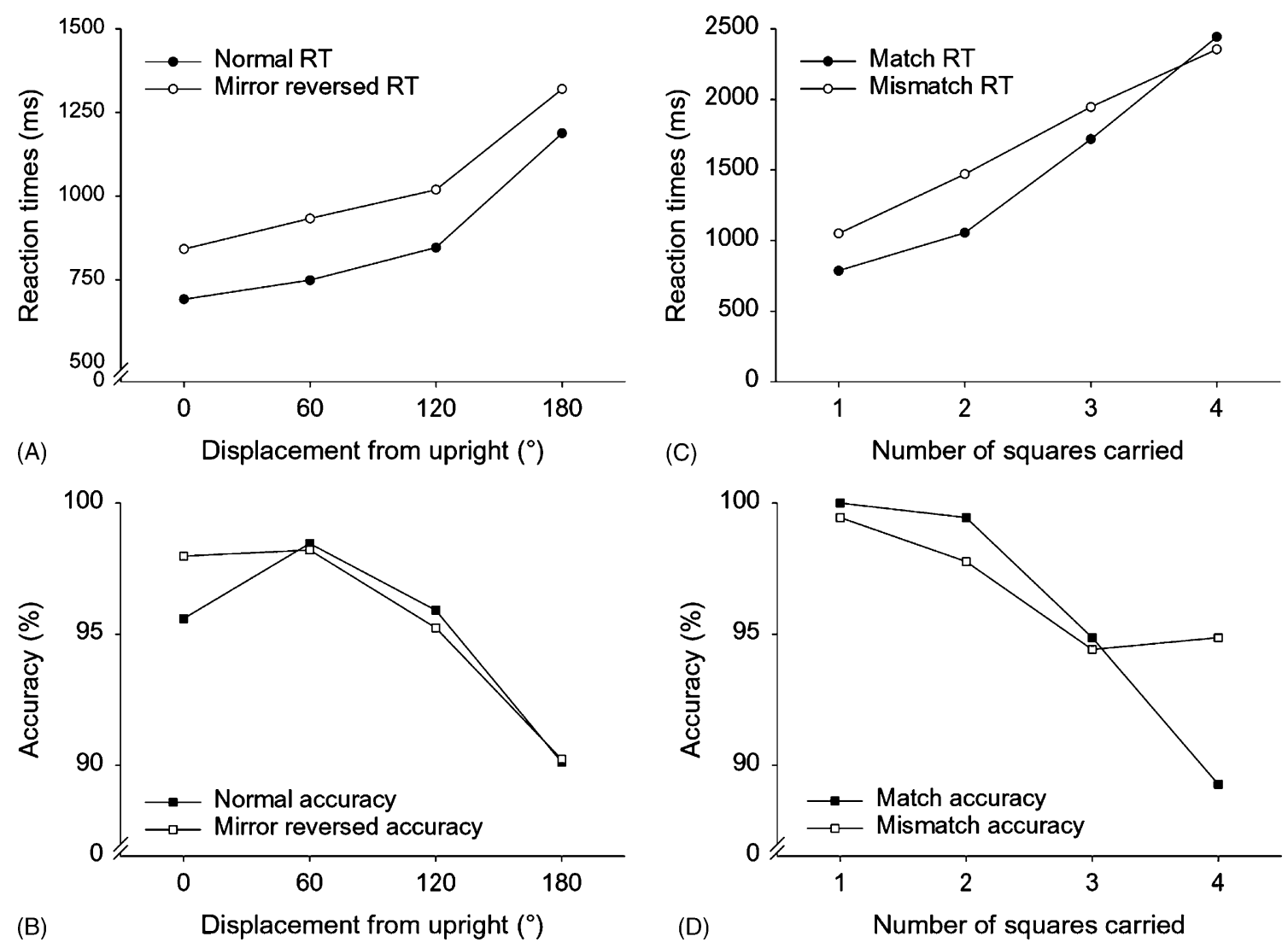

Fig. 2. (A) Grand averaged reaction times and (B) accuracies for mental-rotation task; and (C) reaction times and (D) accuracy for mental paper folding.

reaction times for the minimum and the maximum number of squares carried was $1468 \mathrm{~ms}$. This main effect consisted of a highly significant linear trend $(F(1,13)=62.46$, $P<0.001$ ), which accounted for $99 \%$ of the variance.

There was also a significant interaction between the two main factors $(F(3,39)=6.87, P<0.001)$. The reaction times for both the match and the non-match shapes increased with an increasing number of squares carried, but the increase was greater for the matching shapes than for the non-matching shapes.

There was no significant difference in accuracy between the match and non-match shapes $(F(1,13)<1)$. The average accuracy overall was $96.26 \%$ (Fig. 2(B)). There was a significant effect of the number of squares carried along a fold on accuracy $(F(3,39)=14.80, P<0.001)$. The effect was mainly composed of a linear trend $(F(1,13)=18.82$, $P=0.001)$, explaining $96 \%$ of the variance.

Furthermore there was a significant interaction effect between the two main effects $(F(3,39)=6.03, P=0.002)$. This effect was attributable to the linear $(F(1,13)=8.54$, $P=0.012)$ and quadratic trend component of the number of squares carried $(F(1,13)=7.63, P=0.016)$ which explained 59 and $39 \%$ of the variance, respectively. The accuracy for the matching shapes was lower for the shapes that required 4 squares carried than the accuracy for the shapes that required 1 or 3 squares carried $(P<0.045$ for both, with Bonferroni correction). On the other hand, the accuracy for the 4 squares carried non-match shapes was not significantly different than the accuracy for 1 or 3 squares carried non-match shapes. Furthermore the difference in accuracy between the match and the mismatch shapes is larger at the 4 squares carried level than any other level, with a difference of $5.58 \%$ ( $P=0.028$ with Bonferroni correction).

\subsubsection{Correlation between tasks}

Since both mental-rotation and mental paper-folding tasks are often used as measures of visuospatial ability (e.g. Davison \& Sussman, 2001; Greenfield, Brannon, \& Lohr, 1994) one may expect a given subject to perform similarly on these two tasks. As a final analysis, we examined the relationship between the RT profiles obtained for the two tasks. For each subject, the slope of the function relating reaction time to letter orientation for normal letters in the mental-rotation task, and the slope of the function relating reaction time to the number of squares carried for matching stimuli in the paper-folding task, were computed. The product-moment correlation between these two slopes was not significant $(r=0.215, P>0.05)$.

In summary, the tasks used in the present study resulted in characteristic reaction-time functions; that is, RTs increased monotonically with larger angular displacement of letters up to $180^{\circ}$ for mental rotation (Cooper \& Shepard, 1973), 
and with number of squares carried for mental paper folding (Shepard \& Feng, 1972). The plots in Fig. 2 shows clearly that accuracies decreased as RTs increased, demonstrating that the RT results can not be explained by a speed-accuracy trade-off. The lack of correlation between the two tasks suggests that they may not tap into the identical abilities, and thus may depend on at least partially different neural mechanisms.

\subsection{ERPS}

Following data collection, the EEG files were segmented with respect to event triggers in a $1196 \mathrm{~ms}$ epoch including a $200 \mathrm{~ms}$ pre-stimulus baseline. Voltages were sampled every $4 \mathrm{~ms}$ over this epoch. Only the trials on which the subjects responded correctly were included in the analyses. Eye-movement correction was made on all segments using the method of Jervis, Nichols, Allen, Hudson, and Johnson (1985). The corrected data from each subject were then averaged to produce a total of 8 ERPs for each task (4 orientations $\times 2$ discrimination decisions for rotation, and 4 conditions of number of squares carried $\times 2$ discrimination decisions for paper-folding). In the present report, we retained only the normal letter ERPs for mental rotation, and the match ERP for mental paper folding for further analyses.

Averaged ERPs from individual subjects were grandaveraged, and the grand averages were re-referenced to the average reference. DC offsets were calculated from the pre-stimulus baseline and removed from all waveforms. For all analyses the grand averages were digitally-filtered with a bandpass of $0.1-30 \mathrm{~Hz}$.

Fig. 3 shows the ERPs at the $P_{z}$ electrode for each angle of rotation of normal letters for the mental-rotation task (Fig. 3(A)), and each number of squares carried for matching shapes for the paper-folding task (Fig. 3(B)). The $0^{\circ}$ ERP serves as a non-rotation baseline for each of the mental rotation conditions, and the 1-square carried condition serves as a non-folding baseline for the paper-folding conditions.

Baseline ERPs showed a large-amplitude positive wave extending over a duration of some $400 \mathrm{~ms}$, beginning at around $200 \mathrm{~ms}$ post-stimulus for the mental-rotation task (Fig. 3(A)) and around $300 \mathrm{~ms}$ post-stimulus for the paper-folding task (Fig. 3(B)). The effect of rotation is manifest as a decrease in amplitude of the positive component, an effect which is larger for larger angles of rotation (Fig. 3(A) and (C)). For mental paper folding, a similar amplitude decrease is observed, however, the effect does not seem to increase with larger number of squares carried (Fig. 3(B)). Visual inspection of the data suggests that the effect of mental rotation starts becoming apparent around $380 \mathrm{~ms}$ and ends around $700 \mathrm{~ms}$, while the effect of mental paper folding becomes apparent around $420 \mathrm{~ms}$ and ends around $720 \mathrm{~ms}$.

Since 420-700 ms time period encompasses the effects of both mental rotation and mental paper folding on ERP amplitude, this time period was chosen for further analysis of the
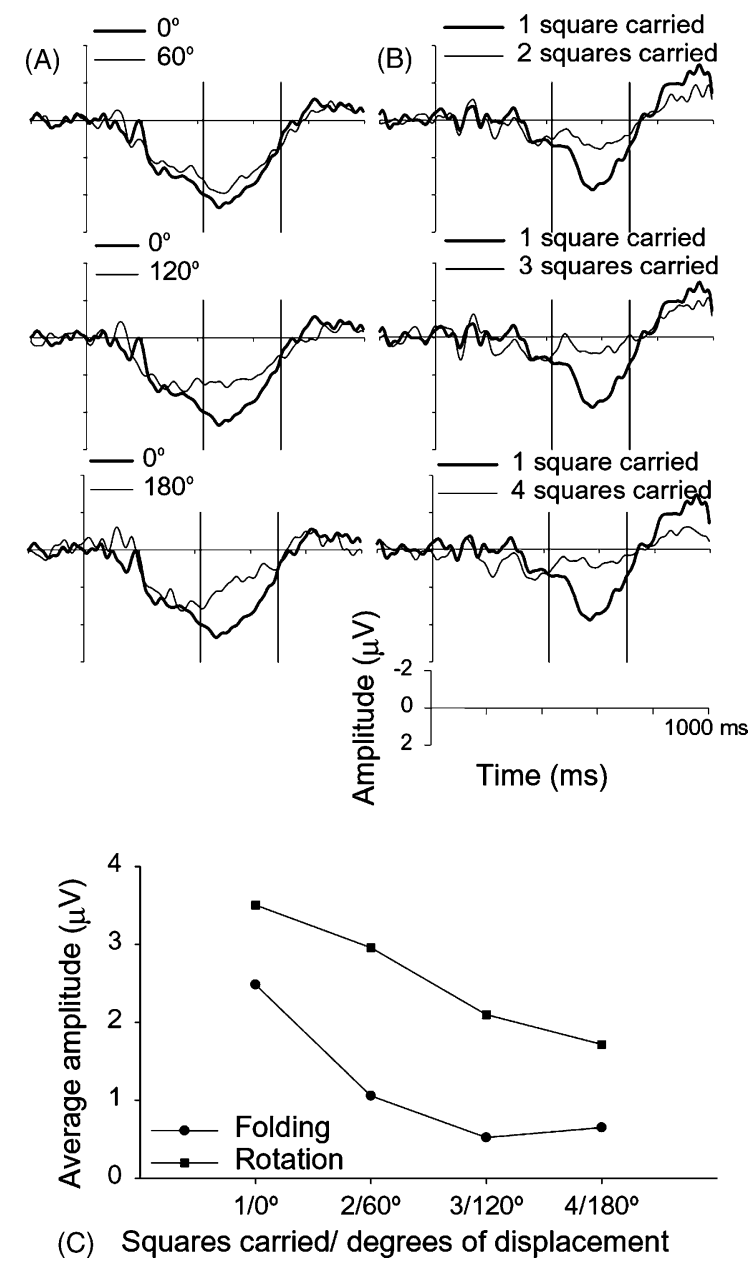

Fig. 3. Grand averaged ERPs from $P_{z}$ electrode for: (A) mental rotation and (B) mental paper folding. The $0^{\circ}$ (non-rotation baseline) ERP and the 1-square carried (non-folding baseline) ERPs are re-plotted against each of the other conditions. Mean amplitude over the 420-700 ms time window for the ERPs from (A) and (B) are plotted in (C).

effect of task difficulty on ERP amplitude change. Fig. 3(C) shows that ERP amplitudes for both tasks are inverse monotonic functions of their respective levels of transformation (angle of required rotation or required number of squares carried). ANOVA for the mental-rotation ERPs showed a highly significant effect of angle of rotation $(F(3,39)=11.90$, $P<0.001)$, with a significant linear trend $(F(1,13)=$ $33.37, P<0.001$ ) which accounted for $98.01 \%$ of the variance. The ERP differences between 0 and $120^{\circ}, 0$ and $180^{\circ}$, 60 and $120^{\circ}$ and 60 and $180^{\circ}$ were significant $(P<0.019)$.

ANOVA for the paper-folding ERPs showed a highly significant effect of number of squares carried $(F(3,39)=$ 19.35, $P<0.001)$, with significant linear $(F(1,13)=$ 29.87, $P<0.001)$ and quadratic $(F(1,13)=45.67$, $P<0.001)$ trends which accounted for 75.06 and $24.84 \%$ of variance, respectively. Differences between 1 square carried and each of the other conditions were significant $(P<0.003$ with Bonferroni correction), but there were no significant differences between the other three conditions. 
In the context of high-density ERP recording, the selection of electrodes for detailed analyses can be problematic because ERP components of interest are typically distributed over many electrodes, and latencies and amplitudes often vary widely over space. Even when a given component is well-localized to a spatial cluster of electrodes, there is typically a polarity-reversed version at a distant location or locations on the head, because of the dipolar nature of the generators of surface ERPs. Thus, a focus on a single electrode or a subset of the total is often unsatisfactory, because it is difficult or impossible to derive a non-arbitrary criterion for the selection. Even worse, this selection defeats the rationale of high-density recording, and may entail the loss of important information. However, in practice, graphical presentation and statistical analyses of 129 channel ERPs is very cumbersome.

One useful solution to the difficulty of dealing with multi-channel data is the Global Field Power (GFP; Lehmann \& Skrandies, 1984) descriptor, which has the advantage of reducing the data from multiple electrodes into a single time series. GFP is calculated as:

$\mathrm{GFP}=\sqrt{\frac{1}{2 n} \times \sum_{i=1}^{n} \sum_{j=1}^{n}\left(u_{i}-u_{j}\right)^{2}}$

where $n$ is the number of electrodes which measure the potentials $e_{i}$ and $e_{j} ; i, j=1 \ldots n$; the observed voltages are $u_{i}=e_{i}-e_{\text {common reference }}$ and describes the degree of global relief (or "hilliness") of the voltage topography at each point in time.

As we were primarily interested in the experimental effects manifest as the difference between a given condition and its baseline, we employed a variant of the GFP calculation which we refer to as "scalp effect strength" (SES), calculated as:

$\mathrm{SES}=f(t)=\frac{\sum_{i=1}^{n}\left(x_{i t}-y_{i t}\right)^{2}}{n}$

where $n$ is the number of electrodes, and $x_{i t}$ and $y_{i t}$ are the two voltages to be compared at electrode $i$ and time $t$.

This description expresses the global magnitude of experimental effect at each point in time. Note that the SES calculation retains the squared values of the differences (where GFP takes the square root of the sum of squared voltages). As we are dealing with differences, and consequently an increase in the amount of noise in the data, we have found it useful to deal with squared values which have the useful property of enhancing the difference between small and large values. Experimental effects are shown in this manner in Fig. 4.

\subsection{Temporal course of experimental effects in mental rotation}

Beginning with the $60^{\circ}$ condition, SES values are near 0 during the pre-stimulus baseline, and first exhibit an increase over the pre-stimulus values just prior to $200 \mathrm{~ms}$, and then show a sustained increase from approximately $300 \mathrm{~ms}$ to approximately $625 \mathrm{~ms}$, after which the SES decreases again to the end of the epoch (but not to pre-stimulus values).

In order to determine whether observed SES peaks were significantly different from 0 , we calculated mean SES amplitudes over $48 \mathrm{~ms}$ time windows for each subject, centered on SES peaks of interest derived from the grand average SES plots. We performed a total of $12 t$-tests, using the Bonferroni correction for multiple comparisons to obtain a critical $P$ of $0.05 / 12=0.00417$.

For the $60^{\circ}$ condition, the early SES peak was significantly different from $0(t(13)=6.73, P<0.0001)$, as was the later SES segment $(t(13)=7.67, P<0.0001)$. (No clear peak was present in this plot, so the analysis time window was centered in the middle of the late, increase at 476-524 ms).

For $120^{\circ} \mathrm{SES}$ values show a similar pattern to approximately $400 \mathrm{~ms}$, thereafter they exhibit a considerably larger increase with a peak amplitude at approximately $480 \mathrm{~ms}$, a second amplitude peak at approximately $524 \mathrm{~ms}$, and a rapid decline thereafter to approximately $700 \mathrm{~ms}$. Both of the SES peaks were significantly different from $0(t=6.61, P<$ 0.0001 , and $t(13)=4.44, P<0.0001$ for the early and late peaks, respectively).

For $180^{\circ}$, SES does not begin the rapid increase until approximately $420 \mathrm{~ms}$, with an amplitude peak at approximately $528 \mathrm{~ms}$, and a sharp but irregular decline thereafter until approximately $700 \mathrm{~ms}$. Both of the SES peaks were significantly different from $0(t(13)=6.24, P<0.0001$, and $t(13)=7.41, P<0.0001$ for the early and late peaks, respectively).

It is difficult to precisely quantify the onsets, offsets and durations of the various components of the SES plots, because they are fairly irregular throughout, and at various points descend close to the value of the pre-stimulus noise levels. Nonetheless these descriptions illustrate several regularities of the data that correspond neatly with the level of the experimental manipulation and with their respective RT functions: (1) there are two primary effects visible at all three levels of stimulus orientation - an earlier one that peaks at approximately $200 \mathrm{~ms}$, and a later one that peaks between 400 and $600 \mathrm{~ms}$; (2) the amplitude of the $400-600 \mathrm{~ms}$ peak increases systematically with angle of orientation; (3) the onsets of the 400-600 ms component increase systematically with angle of orientation; (4) the offsets of the 400-600 ms component increase systematically with angle of orientation.

\subsection{Temporal course of experimental effects in mental paper folding}

Beginning with the 2-squares carried condition, only a late effect is visible in this plot, with an onset of approximately $400 \mathrm{~ms}$, a well-defined amplitude peak occurring at $580 \mathrm{~ms}$, a second amplitude peak at $620 \mathrm{~ms}$, and an offset 

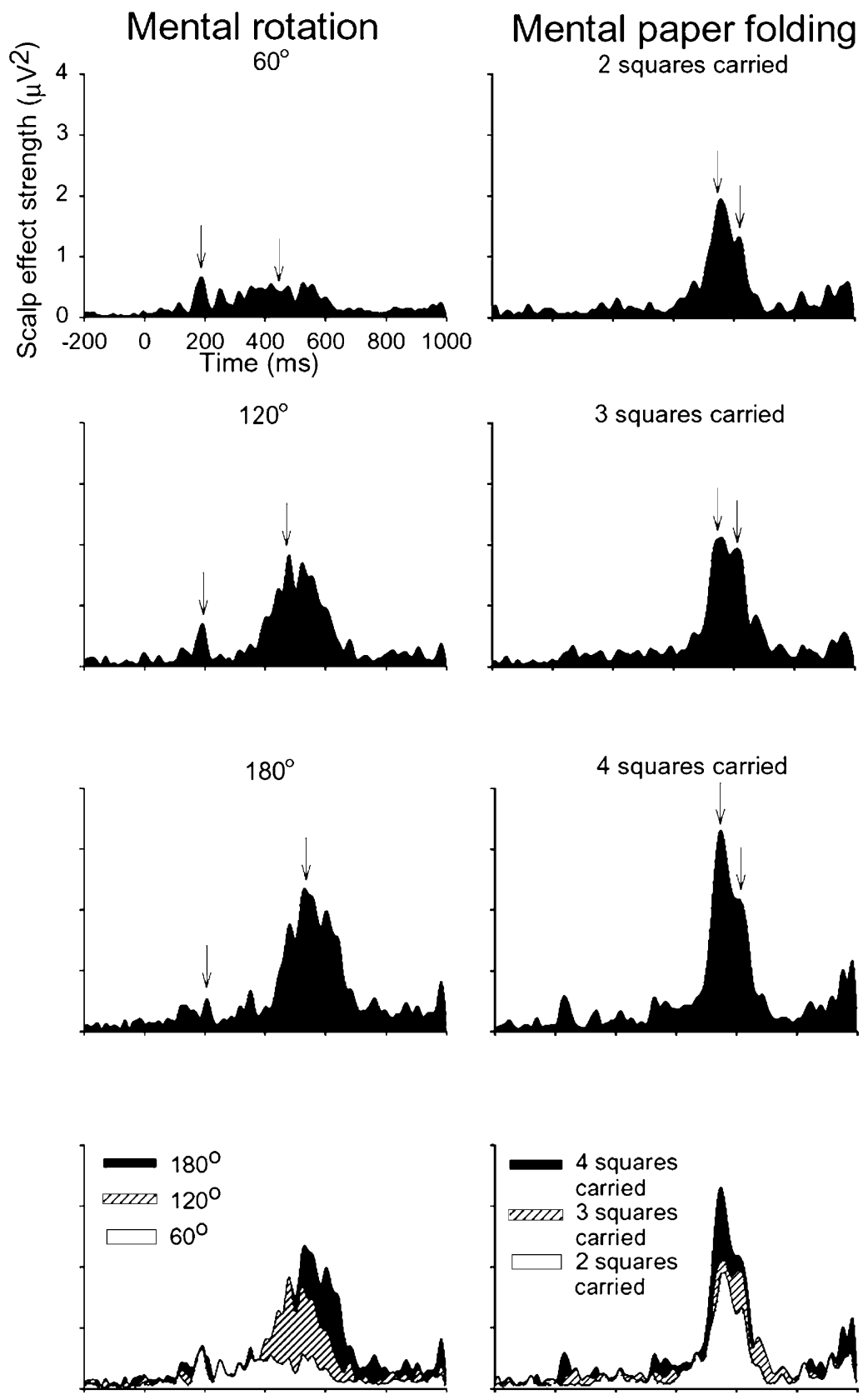

Fig. 4. Scalp effect strength for each level of mental rotation and mental paper folding. Arrows indicate centers of $48 \mathrm{~ms}$ time windows selected for analyses. Bottom plots show superimposition of the upper plots.

at approximately $700 \mathrm{~ms}$. Very similar SES profiles are observed for the other two levels of paper-folding. Indeed, the overlay plot shows that the two amplitude peaks are temporally coincident for all the three conditions, although a systematic increase in amplitude for both peaks as a function of increasing number of squares carried was also observed. Statistical analysis showed that all six of these SES peaks were significantly $>0$ (for the $580 \mathrm{~ms}$ peak: $t(13)=4.90$, $P=0.0003 ; t(13)=5.22, P=0.0002$; and $t(13)=$ $5.27, P=0.0002$, for $2-4$ squares, respectively. For the 620 ms peak: $t(13)=5.08, P=0.0002 ; t(13)=6.02$,
$P<0.0001 ; t(13)=4.69, P=0.0004$ for $2-4$ squares, respectively).

\subsection{Spatial distribution of experimental effects}

As the next step in our analysis, we wished to determine whether these experimental effects exhibited spatial distributions that could be reasonably related to brain generators. Fig. 5 shows mean iso-voltage contour distributions calculated from $48 \mathrm{~ms}$ time windows centered on the SES peaks from the preceding analysis. Fig. 5(A) shows that 
(A)

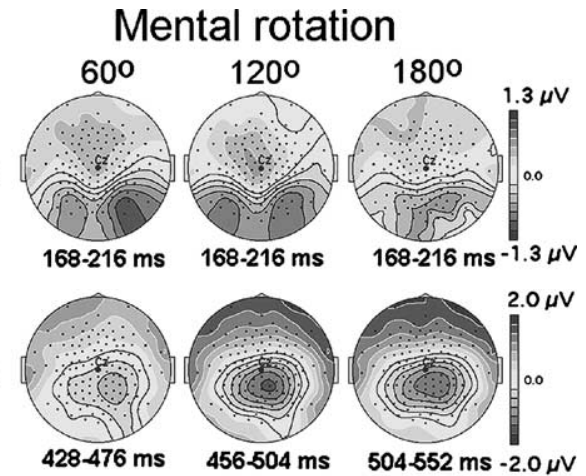

\section{Mental paper folding}

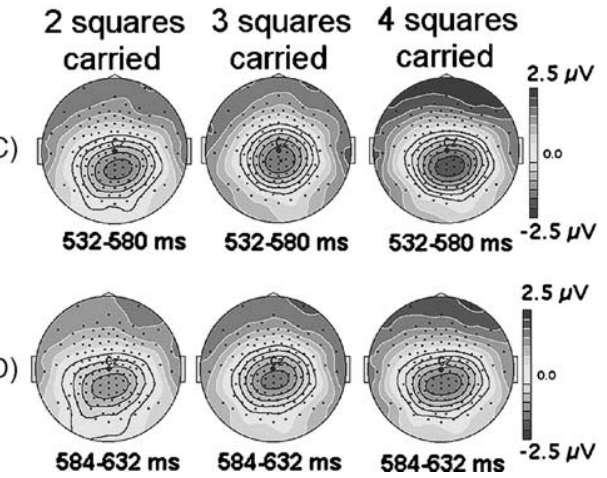

Fig. 5. Iso-voltage contour plots showing spatial distributions of differences from baseline for the time-windows of interest from peaks in the SES plots of Fig. 4. (A) Early occipital peak in mental-rotation task; (B) late parietal peak in mental-rotation task; (C) first parietal peak in paper-folding task; and (D) second parietal peak in paper-folding task.

the early SES peak for mental rotation is distributed over bilateral occipital electrodes for the 60 and $120^{\circ}$ conditions, and over medial occipital electrodes for $180^{\circ}$. Note the corresponding polarity-reversed versions of these fields at fronto-central electrodes.

Fig. 5(B) shows that the late SES peak for mental rotation is distributed over parietal electrodes for all three levels of rotation, with a corresponding polarity-reversed version distributed over anterior temporal, fronto-polar and facial electrodes. The parietal effects are visibly biased to the right hemisphere electrodes in all three conditions.

Fig. 5(C) shows that the first of the late SES peaks for mental paper folding is distributed over parietal electrodes, but with no obvious lateralization. A nearly identical spatial distribution is observed for the second of the late SES peaks for mental paper folding (Fig. 5(D)).

To more formally examine the possible lateralization of the late parietal effect in mental rotation observed in Fig. 5, we collapsed across levels of each task, and calculated the mean voltage over two clusters of 14 parietal electrodes on either side of the midline (Fig. 6). We performed a total of three $t$-tests, using the Bonferonni correction for multiple comparisons to obtain critical $P$ of $0.05 / 3=0.0167$ and found no significant difference between left and right hemi-

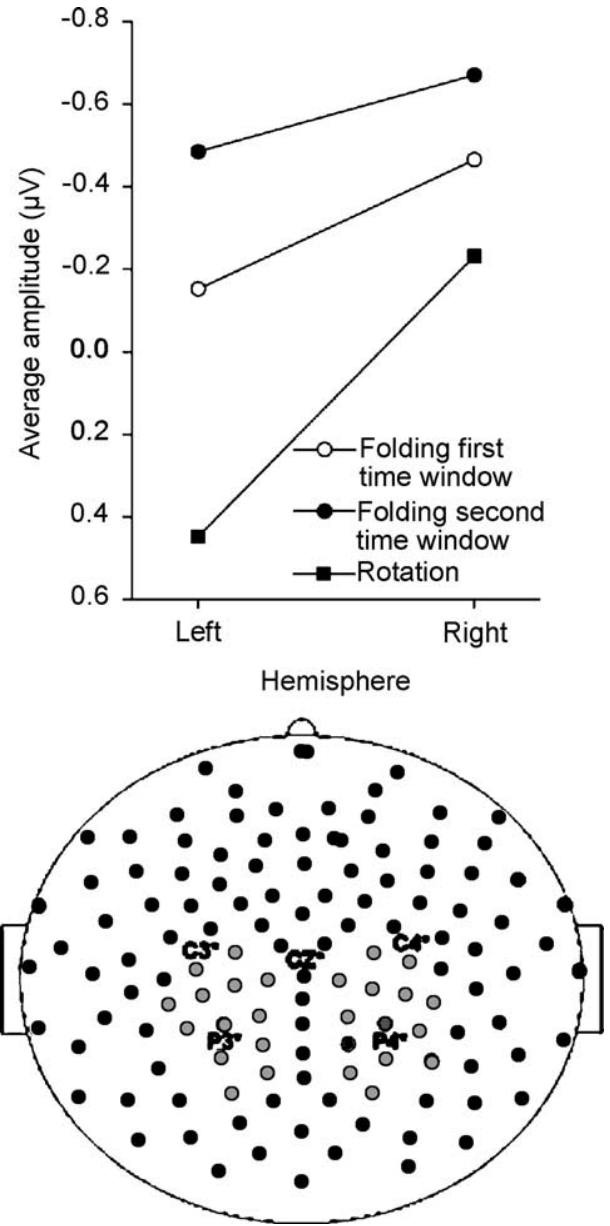

Fig. 6. Parietal lateralization for mental rotation and mental paper-folding tasks. Head diagram at the bottom shows the two subsets of 14 electrodes used in the hemispheric comparisons (grey only) among the full set of electrodes (black and grey).

spheres for both late parietal peaks for mental paper folding $(t(13)=1.37, P=0.19$; and $t(13)=0.82, P=0.42$ for the first and second peaks, respectively). For mental rotation, on the other hand, the right hemisphere showed significantly greater amplitudes than the left hemisphere $(t(13)=4.49$, $P=0.006)$.

\section{Discussion}

The present experiment compared electrophysiological brain responses measured, with a high density of spatial sampling, during two tasks that required subjects to perform mental transformations of visual images: a relatively simple task whose correct performance involved a single, holistic mental transformation (mental rotation of a letter in the picture plane), and a more complicated task whose correct performance clearly demanded a coordinated sequence of mental transformations (paper folds, or mental rotations out of the picture plane). Both tasks resulted in an amplitude modulation of a circa $420-700 \mathrm{~ms}$ ERP 
component distributed over parietal cortex, an effect that was significantly right-lateralized for the single transformation task, but bilateral for the sequential transformation task. In the following, we discussed the functional significance of the ERP amplitude modulation and implications for our understanding of the brain processes that support mental image transformations and those that differentiate the two mental-rotation tasks.

\subsection{Functional significance of the ERP amplitude modulation}

Amplitude modulation of the circa $400-700 \mathrm{~ms}$ parietal ERP component during mental rotation of letters was initially described in 1989 by Peronnet and Farah (1989) and Wijers, Otten, Feenstra, Mulder, and Mulder (1989), and the basic phenomenon has been extensively replicated since then. Wijers et al. (1989) interpreted this amplitude effect as a slow parietal negative wave superimposed upon a temporally- and spatially-coincidents, but functionally independent, P300 complex. As seen in the present results, the amplitude of the slow parietal negativity increases monotonically as a function of the angular disparity of stimuli from the upright position, mirroring the RT results and suggesting that this ERP component is closely tied to the neurophysiological operations underlying mental rotation. This inference has been strongly validated in a series of experiments by Heil, who have systematically elucidated the functional and temporal characteristics of this component (see Heil, 2002 for a review).

Taken together, the studies of Heil (2002) and Heil et al. (1998) provide compelling evidence for the hypothesis that the parietal amplitude modulation is an electrophysiological marker for the orientation-normalization process referred to as "mental rotation." There remains, however, a fundamental puzzle concerning this functional relationship: it is unclear how a mental process that results in behavioral latency changes could be mediated by a neurophysiological process that is manifest only as simple amplitude changes. Put another way, if mental rotations are really analogous to physical rotations, we would logically expect that larger rotations would result in longer durations of activation of the neuronal substrates, rather than simply larger amounts of activation.

Note that all of the mental rotation studies described above analyzed data only from a single parietal electrode or a few electrodes in the parietal region. ERP duration effects do emerge if the analysis incorporates data from many more recording channels. Pegna et al. (1997) applying a spatio-temporal segmentation technique to global field power summaries of 51-channel ERP data obtained in a mental letter-rotation task, found that the duration of a post $-400 \mathrm{~ms}$ component increased systematically with increasing angular disparity from the upright. Our SES analysis, which summarizes the magnitude of an experimental effect over all recording channels, shows both an amplitude effect and a duration effect. Thus, the present results, taken together with those of Pegna et al., indicate that a more accurate characterization of the electrophysiological consequences of mental rotation will require the analysis of data from multiple and spatially-distributed recording electrodes. Further research is clearly required to refine the application of integrative multichannel analytic techniques, such as GFP and SES, to the study of mental rotation.

While SES analysis also showed an orientation-dependent amplitude effect for an early (approximately $200 \mathrm{~ms}$ latency) component, this effect had an occipital distribution and was considerably smaller in amplitude for $180^{\circ}$ than for the other two orientations. This early occipital effect is likely due to fairly low-level processing of orientation by cells in visual cortex.

Mental paper folding ERPs showed no early occipital effect, but showed a clear late parietal effect during a nearly identical time window to that observed for mental letter rotation, a result that indicates that the mental transformations involved draw on a broadly similar class of neural computations. In fact, we have assumed throughout that the mental paper folding is a kind of mental rotation, but while the letter-rotation task involved a single holistic rotation through angles ranging from 60 to $180^{\circ}$, the paper-folding task required subjects to perform a coordinated sequence of rotations through a constant angular distance of $90^{\circ}$. Concordant with this interpretation, the SES time series obtained for 2-4 squares carried all show amplitude peaks that are tightly aligned in time, with no evidence for changes in latency or duration as was the case for mental letter rotation. We note also that the $P_{z}$ amplitude modulation (the standard electrophysiological measure of mental rotation) did not increase significantly as the number of squares carried increased after two. Taken together, these results indicate that the ERP amplitude modulation observed in the first $1000 \mathrm{~ms}$ after stimulus onset in the mental paper-folding task indexes the mental rotation required to make the first $90^{\circ}$ fold in each of the sequences of $2-4$-folds. (This interpretation predicts that we should be able to observe later amplitude modulations corresponding to the subsequent folds, and we have examined this possibility in longer segments of the same data. Unfortunately, because brain processes become progressively less synchronized to experimental events with increasing temporal distance from the events, the signal-to-noise ratio of the post 1-s segments was too low to permit detection of event-related potentials.)

On the other hand, the SES time series for mental paper folding do show a systematic increase in amplitude with increasing number of squares carried. From the present results it is unclear how to reconcile this discrepancy between the $P_{z}$ amplitude modulation and the SES amplitude modulation: in the case of letter rotation, both analyses showed a systematic amplitude modulation with increasing angular disparity. However, in the latter case, we have already demonstrated that SES extracts information (duration) that is not evident in the single channel data, and the mental paper folding results indicate that the SES amplitude modulation may index 
more subtle neuronal processes than are detected in the single channel analysis. One obvious possibility is that larger populations of neurons are recruited to perform the mental rotation as the task becomes more difficult. In the present context, the tasks did not simply take longer to perform as subjects were required to mentally rotate through larger angular distances or to carry larger numbers of squares, but they also become more difficult to perform, with a consequent decline in accuracy. This question clearly merits further investigation with SES measures.

\subsection{Hemispheric lateralization}

Corballis (1997) has pointed out that, as a cognitive process, mental rotation is in many ways comparable to language. Like language, mental rotation is a relatively high-level ability that emerges during cognitive development and involves transformations of mental representations. However, unlike language, mental rotation is non-symbolic, and non-computational; rather, its properties suggest that it is implemented in a fundamentally different manner, as a continuous, analogue process, operating on representations that resemble templates. As language is well known to be left-lateralized, the contrasting properties of mental rotation provide the a priori grounds for a long-held supposition that it may be oppositely lateralized to the right cerebral hemisphere. As we have indicated in Section 1, the empirical evidence for this supposition is currently equivocal: while a number of neuroimaging and neuropsychological studies have reported evidence for a right hemispheric superiority for mental rotation, others have reported evidence for bilateral involvement or even a left-hemisphere superiority. The existing electrophysiological evidence is similarly inconclusive: while several studies have reported a right parietal bias for the orientation-dependent ERP amplitude effect, a number of others have reported no significant lateralization. However, the low-density recording montages employed in these studies achieve a very sparse sampling of the surface electrical fields and consequently offer relatively little precision when considering questions of hemispheric lateralization.

The high-density recordings of the present study provide the strongest electrophysiological evidence to date that mental rotation of letters is a right-parietal dominant function, a result that is concordant with the results of a recent PET neuroimaging study using a similar mental letter-rotation paradigm (Harris et al., 2000). However, our results also show that when the same subjects performed the more complex and sequential transformations involved in mental paper folding, the parietal ERP amplitude modulation showed no significant lateralization, indicating that both the hemispheres were involved to an approximately equal extent. These results are consistent with evidence that the left-hemisphere is specialized for sequential organization and part-wise decomposition of shapes (Corballis, 1991) and may become increasingly engaged in spatial per- formance as the complexity of the task increases (De Renzi, 1978; McGuinness \& Bartell, 1982).

Taken together, the ERP results for the two mental transformation tasks suggest that, while there may be an overall right hemisphere superiority for mental rotation, this bias is not absolute. As described in Section 1, studies of a commissurotomy patient show that he was eventually able to acquire the ability to perform mental rotation in the left hemisphere (Corballis \& Sergent, 1989). Thus, it seems clear that mental rotation can be implemented in both hemispheres. The present results indicate that the right hemisphere may be preferentially engaged when the task is simple and involves a single transformation, but that the left hemisphere is also engaged as the task becomes more complex, as when a coordinated sequence of transformations are required. This task-dependent lability may well account for some of the inconsistent and even contradictory results obtained in previous neuroimaging and electrophysiological studies of hemispheric lateralization for mental rotation.

\section{Acknowledgements}

This work was supported by the Royal Society of New Zealand Marsden Fund Grant UOA813. We thank the editor and two anonymous reviewers for helpful comments on an earlier version of this article.

\section{References}

Alivisatos, B., \& Petrides, M. (1997). Functional activation of the human brain during mental rotation. Neuropsychologia, 35, 111-118.

Bethell-Fox, C., \& Shepard, R. N. (1988). Mental rotation: Effects of complexity and familiarity. Journal of Experimental Psychology: Human Perception and Performance, 14, 12-23.

Cooper, L. A., \& Shepard, R. N. (1973). Chronometric studies of the rotation of mental images. In W. G. Chase (Ed.), Visual information processing (pp. 175-176). New York: Academic Press.

Cooper, L. A., \& Shepard, R. N. (1975). Mental transformations in the identification of left and right hands. Journal of Experimental Psychology: Human Perception and Performance, 104(1), 48-56.

Corballis, M. C. (1991). The lopsided ape. New York: Oxford University Press.

Corballis, M. C. (1997). Mental rotation and the right hemisphere. Brain and Language, 57, 100-121.

Corballis, M. C., \& Sergent, J. (1989). Mental rotation in a commissurotomized patient. Neuropsychologia, 27, 585-597.

Corballis, M. C., Zbrodoff, N. J., Shetzer, L. I., \& Butler, P. B. (1978). Decisions about identity and orientation of rotated letters and digits. Memory and Cognition, 6, 98-107.

Davison, K. K., \& Sussman, E. (2001). Are hormone levels and cognitive ability related during early adolescence? International Journal of Behavioral Development, 25, 416-428.

De Renzi, E. (1978). Hemispheric asymmetry as evidenced by spatial disorders. In M. Kinsbourne (Ed.), Asymmetrical function of the human brain (pp. 49-85). New York: Cambridge University Press.

Ely, M. G. (1982). Identifying rotated letter-like symbols. Memory and Cognition, 10, 25-32.

Farah, M. J., \& Hammond, K. M. (1988). Mental rotation and orientation-invariant object recognition: Dissociable processes. Cognition, 29, 29-46. 
Ferree, T. C., Luu, P., Russell, G. S., \& Tucker, D. M. (2001). Scalp electrode impedance, infection risk, and EEG data quality. Clinical Neurophysiology, 112, 536-544.

Georgopoulos, A. P. (1995). Motor cortex and cognitive processing. In M. S. Gazzanaga (Ed.), The cognitive neurosciences (pp. 507-517). Cambridge: MIT Press.

Georgopoulos, A. P., Lurito, J. T., Petrides, M., Schwartz, A. B., \& Massey, J. T. (1989). Mental rotation of the neuronal population vector. Science, 241, 234-236.

Georgopoulos, A. P., \& Pellizzer, G. (1995). The mental and the neural: Psychological and neural studies of mental rotation and memory scanning. Neuropsychologia, 33(11), 1531-1547.

Georgopoulos, A. P., Taira, M., \& Lukashin, A. (1993). Cognitive neurophysiology of the motor cortex. Science, 260, 47-52.

Greenfield, P. M., Brannon, G., \& Lohr, D. (1994). Two-dimensional representation of movement through three-dimensional space: The role of video game expertise. Journal of Applied Developmental Psychology, $15,87-103$.

Hamm, J. P. (2001). Object oriented timers for the PC. Behavior Research Methods, Instruments and Computers, 33, 532-539.

Harris, I. M., Egan, G. F., Sonkkila, C., Tochon-Danguy, H. J., Paxinos, G., \& Watson, J. D. G. (2000). Selective right parietal lobe activation during mental rotation: A parametric PET study. Brain, 123, 65-73.

Heathcote, A. (1988). Screen control and timing routines for the IBM microcomputer family using a high-level language. Behavior Research Methods, Instruments and Computers, 20, 289-297.

Heil, M. (2002). The functional significance of ERP effects during mental rotation. Psychophysiology, 39(5), 535-545.

Heil, M., Rauch, M., \& Hennighausen, E. (1998). Response preparation begins before mental rotation is finished: Evidence from event-related brain potentials. Acta Psychologia, 99, 217-232.

Jasper, H. H. (1958). The Ten-Twenty Electrode System of the International Federation. Electroencephalography and Clinical Neurophysiology, 10, 371-375.

Jervis, B. W., Nichols, M. J., Allen, E. M., Hudson, N. R., \& Johnson, T. E. (1985). The assessment of two methods for removing eye movement artefact from the EEG. Electroencephalography and Clinical Neurophysiology, 61, 444-452.

Johnson, B. W., \& Hamm, J. P. (2000). High-density mapping in a N400 paradigm: Evidence for bilateral temporal lobe generators. Clinical Neurophysiology, 111, 532-545.

Jolicoeur, P. (1985). The time to name disoriented objects. Memory and Cognition, 13, 289-303.

Jolicoeur, P. (1990). Identification and disoriented objects: A dual systems theory. Mind and Language, 5, 387-410.

Just, M. A., \& Carpenter, P. A. (1985). Cognitive coordinate systems: Accounts of mental rotation and individual differences in spatial ability. Psychological Review, 92, 137-172.
Kosslyn, S. M., Digirolamo, G. J., Thompson, W. L., \& Alpert, N. M. (1998). Mental rotation of objects versus hands-neural mechanisms revealed by positron emission tomography. Psychophysiology, 35, 151161.

Lehmann, D., \& Skrandies, W. (1984). Spatial analysis of evoked potentials in man-a review. Progress in Neurobiology, 23, 227250.

McGuinness, D., \& Bartell, T. E. (1982). Lateral asymmetry: Hard or simple-minded? Neuropsychologia, 20, 629-639.

Mehta, Z., \& Newcombe, F. (1991). A role for the left hemisphere in spatial processing. Cortex, 27, 151-167.

Murray, J. E. (1997). Flipping and spinning: Spatial transformation procedures in the identification of rotated natural objects. Memory and Cognition, 25(1), 96-105.

Oldfield, R. C. (1971). The assessment and analysis of handedness: The Edinburgh Inventory. Neuropsychologia, 9, 97-113.

Pegna, A. J., Khateb, A., Spinelli, L., Seeck, M., Landis, T., \& Michel, C. M. (1997). Unraveling the cerebral dynamics of mental imagery. Human Brain Mapping, 5, 410-421.

Peronnet, F., \& Farah, M. J. (1989). Mental rotation: An event-related potential study with a validated mental-rotation task. Brain and Cognition, 9, 279-288

Shepard, R. N., \& Feng, C. (1972). A chronometric study of mental paper folding. Cognitive Psychology, 3, 228-243.

Shepard, R. N., \& Metzler, J. (1971). Mental rotation of three-dimensional objects. Science, 171, 701-703.

Stuss, D. T., Sarazin, F. F., Leech, E. E., \& Picton, T. W. (1983). Event-related potentials during naming and mental rotation. Electroencephalography and Clinical Neurophysiology, 56, 133146

Tagaris, G. A., Kim, S. -G., Strupp, J. P., Anderson, P., Ugurbil, K., \& Georgopoulos, A. (1996). Quantitative relations between parietal activation and performance in mental rotation. NeuroReport, 7, 773776.

Tagaris, G. A., Kim, S. -G., Strupp, J. P., Andersen, P., Ugurbil, K., \& Georgopoulos, A. P. (1997). Mental rotation studied by functional magnetic resonance imaging at high field (4 T): Performance and cortical activation. Journal of Cognitive Neuroscience, 9, 419432.

Tucker, D. M. (1993). Spatial sampling of head electrical fields: The geodesic sensor net. Electroencephalography and Clinical Neurophysiology, 87, 154-163.

White, M. J. (1980). Naming and categorization of tilted alphanumeric characters do not require mental rotation. Bulletin of the Psychonomic Society, 15, 153-156.

Wijers, A. A., Otten, L. J., Feenstra, S., Mulder, G., \& Mulder, L. J. M. (1989). Brain potentials during selective attention, memory search, and mental rotation. Psychophysiology, 26(4), 452-467. 